\title{
Tame multiplicity and conductor for local Galois representations
}

\author{
Colin J. Bushnell and Guy Henniart \\ King's College London and Université de Paris-Sud
}

July 2018, revised April 2019

\begin{abstract}
Let $F$ be a non-Archimedean locally compact field of residual characteristic $p$. Let $\sigma$ be an irreducible smooth representation of the absolute Weil group $\mathcal{W}_{F}$ of $F$ and $\operatorname{sw}(\sigma)$ the Swan exponent of $\sigma$. Assume $\operatorname{sw}(\sigma) \geqslant 1$. Let $\mathcal{J}_{F}$ be the inertia subgroup of $\mathcal{W}_{F}$ and $\mathcal{P}_{F}$ the wild inertia subgroup. There is an essentially unique, finite, cyclic group $\Sigma$, of order prime to $p$, so that $\sigma\left(\mathcal{I}_{F}\right)=\Sigma \sigma\left(\mathcal{P}_{F}\right)$. In response to a query of Mark Reeder, we show that the multiplicity in $\sigma$ of any character of $\Sigma$ is bounded by $\operatorname{sw}(\sigma)$.
\end{abstract}

\section{CONTEnTS}

1. Introduction

2. Group-theoretic preliminaries

3. Conductor estimate for primitive representations

4. Certain primitive representations

5. An estimate of the different

6. Proof of the main theorem

\section{INTRODUCTION}

1.1. Let $F$ be a non-Archimedean, locally compact field of residual characteristic $p$. Let $\bar{F}$ be a separable algebraic closure of $F$ and $\mathcal{W}_{F}$ the Weil group of $\bar{F} / F$. Write $\mathcal{J}_{F}$ for the inertia subgroup of $\mathcal{W}_{F}$ and $\mathcal{P}_{F}$ for the wild inertia subgroup.

2000 Mathematics Subject Classification. 11S37, 11S15, 22E50.

Key words and phrases. Local field, tame multiplicity, conductor bound, primitive representation. 
Let $\sigma$ be an irreducible, smooth, complex representation of $\mathcal{W}_{F}$. Thus $I=$ $\sigma\left(\mathcal{J}_{F}\right)$ and $P=\sigma\left(\mathcal{P}_{F}\right)$ are finite groups, with $P$ being the unique $p$-Sylow subgroup of $I$. The quotient $I / P$ is cyclic, of order prime to $p$. It follows readily that there is a subgroup $\Sigma$ of $P$ such that the quotient map $I \rightarrow I / P$ induces an isomorphism $\Sigma \rightarrow I / P$. Thus $\Sigma \cap P=1$ and $I=\Sigma P$. Moreover, the subgroup $\Sigma$, satisfying these conditions, is uniquely determined up to conjugation by an element of $P$. (See, for instance, [6] Ch. 6 Th. 4.1, for a full discussion.) Define the tame multiplicity $m(\sigma)$ of $\sigma$ by

$$
m(\sigma)=\max _{\chi} \operatorname{dim} \operatorname{Hom}_{\Sigma}(\chi, \sigma)
$$

where $\chi$ ranges over the group $\widehat{\Sigma}$ of linear characters of $\Sigma$. The integer $m(\sigma)$ does not depend on the choice of $\Sigma$ and, in all cases, $m(\sigma) \geqslant 1$.

Let $\operatorname{sw}(\sigma)$ be the Swan exponent of $\sigma$. We prove:

Tame Multiplicity Theorem. Let $\sigma$ be an irreducible smooth representation of $\mathcal{W}_{F}$. If $\operatorname{sw}(\sigma)>0$, then

$$
m(\sigma) \leqslant \operatorname{sw}(\sigma)
$$

In particular, the space of $\Sigma$-fixed points in $\sigma$ has dimension at most $\operatorname{sw}(\sigma)$.

In his paper [10], Mark Reeder gives compelling reasons for being interested in the invariant $m(\sigma)$ and the inequality (1.1.1). He proves the theorem when $\sigma$ is either essentially tame or of epipelagic type, in the sense that $\operatorname{sw}(\sigma)=1$. This paper is written in response to his query as to whether it might hold in general.

Remarks. A couple of cases can be dispatched straightaway.

(1) If $\operatorname{sw}(\sigma)=0$, then $\Sigma=I$ and $\sigma$ is induced from a tamely ramified character of $\mathcal{W}_{K}$, where $K / F$ is unramified. It follows that $m(\sigma)=1$.

(2) If $\operatorname{dim} \sigma=1$ and $\operatorname{sw}(\sigma) \geqslant 1$, then $m(\sigma)=1$ for trivial reasons.

1.2. This is, obviously, a "small conductor" problem: certainly $m(\sigma) \leqslant \operatorname{dim} \sigma$ while, for the vast majority of representations $\sigma$, one has $\operatorname{dim} \sigma<\operatorname{sw}(\sigma)$. On the other hand, if $\operatorname{sw}(\sigma)=1$ then $m(\sigma)=1[2]$, [10]. It is the contrast between these two extremes that dictates the flavour of the paper. In many cases, rather coarse estimates should suffice to give the result but, in others, delicacy is likely to be required. 
The small-conductor aspect suggests that primitive representations $\sigma$ must play a central rôle. At first glance, one might hope to prove the theorem for primitive representations and then proceed by induction. That light-hearted approach falls at the first hurdle. If one tries to calculate $m(\sigma)$ directly from the description of $\sigma$ in Koch's structure theory [9], the combinatorics rapidly get out of hand. Further, we have an uncertain grasp of the relation between Koch's description of $\sigma$ and the value of $\operatorname{sw}(\sigma)$. Examples show that there is sometimes no room for any sloppiness in the estimates.

More positively, there is a strong lower bound for $\operatorname{sw}(\sigma)$ in [7]. On the other side, help comes from a rather different source. Glauberman's general theory of character correspondences for finite groups [5], as developed in Isaacs' book [8], leads to an exact and manageable formula for $m(\sigma)$, but only for a restricted class of primitive representations $\sigma$. To outline this, we need some terminology.

Let $\tau$ be an irreducible representation of $\mathcal{W}_{F}$. Say that $\tau$ is absolutely ramified if it factors through $\operatorname{Gal}(E / F)$, where $E / F$ is a finite, totally ramified field extension. Let $\sigma$ be primitive and absolutely ramified, viewed as a faithful representation of $G=\operatorname{Gal}(E / F)$. Let $\operatorname{Gal}(E / K)$ be the centre of $G$ and let $T / F$ be the maximal tame sub-extension of $E / F$. We may reduce to the case where $\operatorname{Gal}(E / T)$ is a $p$-group, and therefore the wild inertia subgroup of $G$. Let $\Sigma$ be a complement of $\operatorname{Gal}(E / T)$ in $G$. For the purposes of this introduction, say that $\sigma$ is $\Sigma$-homogeneous if the $G$-centralizer of any non-trivial element of $\Sigma$ is $\Sigma$.Gal $(E / K)$. If $\sigma$ is $\Sigma$-homogeneous, then [8] gives an exact formula for the character $\operatorname{tr} \sigma \mid \Sigma$.

If $\sigma$ is absolutely ramified and $\Sigma$-homogeneous, comparison of the character formula with the conductor bound of [7] yields the theorem. This is a case in which $m(\sigma)$ can be close to $\operatorname{sw}(\sigma)$ (see 4.5). More generally, an absolutely ramified primitive representation is essentially a tensor product of homogeneous ones. A relatively relaxed estimate then gives the theorem in this case.

For the third step, we prove the theorem when $\sigma$ is absolutely ramified (but not necessarily primitive). We can assume that $\sigma$ is an induced representation $\operatorname{Ind}_{K / F} \tau$, where $\tau$ is an absolutely ramified representation of $\mathcal{W}_{K}$ with $K \neq F$ and $m(\tau) \leqslant \operatorname{sw}(\tau)$. A standard property asserts that $\operatorname{sw}(\sigma)=$ $\operatorname{sw}(\tau)+w_{K / F} \operatorname{dim} \tau$, where $w_{K / F}$ is the wild exponent of the extension $K / F$. The relation between $m(\sigma)$ and $m(\tau)$ is group-theoretic in nature, so we have to estimate the arithmetic quantity $w_{K / F}$ in group-theoretic terms. A rather coarse argument suffices. It shows that, relative to induction of representations, $\operatorname{sw}(\sigma)$ grows much more quickly that $m(\sigma)$ and so justifies the initial emphasis 
on primitive representations. From there on, the general case of the theorem follows easily.

1.3. The paper is arranged as follows. The necessary material from finite group theory is assembled in section 2. In section 3, we review some properties of

primitive representations leading to the conductor estimate of [7] Théorème 1.8. We give a complete proof of that result. It uses the same ideas as [7] but, in the present limited context, they can be expressed more succinctly and transparently. Section 4 is the heart of the argument, proving the theorem for " $\Sigma$-homogeneous", absolutely ramified, primitive representations, as sketched above. Section 5 is the group-theoretic estimate of the wild exponent, and section 6 completes the proof.

Acknowledgement. We thank the referee for his detailed comments on an earlier version. These led us to produce a much improved version. In particular, he noticed the elementary error corrected in 2.1 below.

\section{Group-Theoretic Preliminaries}

We gather some techniques from the representation theory of finite groups. This section has its own scheme of notation.

2.1. We consider a special class of finite $p$-groups, using the terminology of [4].

Definition. Let $P$ be a finite $p$-group with centre $Z \neq P$. It is called $H$-cyclic if it satisfies the following conditions.

(1) The centre $Z$ is cyclic, and

(2) the quotient $V=P / Z$ is elementary abelian.

For convenience, we summarize the main properties of these groups, following the account in [4]. For $x, y \in P$, the commutator $[x, y]$ lies in the centre $Z$ and satisfies $[x, y]^{p}=\left[x^{p}, y\right]=1$.

We think of the elementary abelian $p$-group $V$ as a vector space over the field $\mathbb{F}_{p}$ of $p$ elements. Let $\zeta$ be a faithful character of $Z$. The commutator pairing

$$
(x, y) \longmapsto \zeta\left(x y x^{-1} y^{-1}\right), \quad x, y \in P,
$$

takes its values in the group $\boldsymbol{\mu}_{p}(\mathbb{C})$ of complex $p$-th roots of unity. Composing with a fixed isomorphism $\boldsymbol{\mu}_{p}(\mathbb{C}) \rightarrow \mathbb{F}_{p}$, the pairing (2.1.1) induces an alternating bilinear form

$$
h_{\zeta}: V \times V \longrightarrow \mathbb{F}_{p}
$$


Because $Z$ is the centre of $P$, the form $h_{\zeta}$ is nondegenerate. Consequently, $V$ has $p^{2 r}$ elements, for an integer $r \geqslant 1$.

A subspace $W$ of $V$ is a Lagrangian subspace of the alternating space $\left(V, h_{\zeta}\right)$ if it has exactly $p^{r}$ elements and $h_{\zeta}\left(w_{1}, w_{2}\right)=0$ for all $w_{1}, w_{2} \in W$.

Lemma. There is a unique irreducible representation $\tau$ of $P$ such that $\tau \mid Z$ contains $\zeta$. It has the following properties.

(1) The representation $\tau$ is faithful, it satisfies $\operatorname{dim} \tau=p^{r}$, and $\tau \mid Z$ is a multiple of $\zeta$.

(2) Let $W$ be a Lagrangian subspace of $\left(V, h_{\zeta}\right)$ with inverse image $\widetilde{W}$ in $P$. The group $\widetilde{W}$ is abelian and the character $\zeta$ of $Z$ admits extension to a character $\zeta^{W}$ of $\widetilde{W}$. For any such $\zeta^{W}$, one has

$$
\tau \cong \operatorname{Ind}_{\widetilde{W}}^{P} \zeta^{W} .
$$

Proof. See [4] 8.1 Proposition.

Remark. A finite $p$-group is called extra special of class 2 if it is H-cyclic and its centre has order $p$ ( $c f$. [6] p. 183). More generally, let $P$ be $\mathrm{H}$-cyclic with centre $Z$. Since the representation $\tau$ of the lemma is faithful, one may identify $P$ with $\tau(P)$. One can then follow Rigby's argument in [11] Theorem 2 to show that $P$ is the central product of the finite cyclic $p$-group $Z$ and an extra special $p$-group of class 2 .

Corrigendum. In the preamble to [4] 8.1, we assert that an H-cyclic group is extra special of class 2 . The arguments of [4] section 8 are conducted axiomatically, so this error has no effect on the results or their proofs. In particular, the lemma above remains valid.

2.2. Let $P$ be a finite, $\mathrm{H}$-cyclic $p$-group with centre $Z$, write $V=P / Z$ and let $|V|=p^{2 r}$. Let $\zeta$ be a faithful character of $Z$. We introduce another element of structure.

Definition. Let $S$ be a cyclic group of automorphisms of $P$, such that

(1) the order $|S|$ of $S$ is not divisible by $p$ and

(2) $S$ acts trivially on $Z$.

Because of condition (2), the action of $S$ on $P$ fixes the commutator pairing

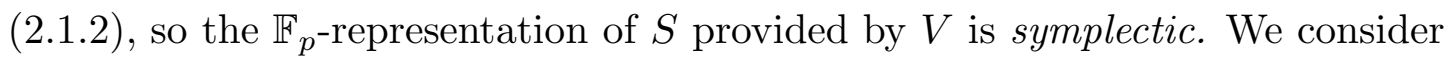
a specific representation of the semi-direct product $G=S \ltimes P$. 
Lemma. Let $G=S \ltimes P$ and let $\tau$ be the unique irreducible representation of $G$ such that $\tau \mid Z$ contains $\zeta$.

(1) There exists a unique representation $\tilde{\tau}$ of $G$ such that $\tilde{\tau} \mid P \cong \tau$ and $\operatorname{det} \tilde{\tau}(s)=1$, for all $s \in S$.

(2) An irreducible representation $\rho$ of $G$ satisfies $\rho \mid P \cong \tau$ if and only if there is a character $\chi$ of $S=G / P$ such that $\rho \cong \chi \otimes \tilde{\tau}$.

Proof. This is a pleasant exercise, written out in [1] (8.4.1) Proposition. For a very general result of this kind, see [8] 13.3 Lemma.

Under certain circumstances, one can write down the character $\operatorname{tr} \tilde{\tau}$ of $\tilde{\tau}$ on elements of $S$.

Proposition. Suppose that, for every $s \in S, s \neq 1$, the $G$-centralizer of $s$ is $S Z$. There is then a constant $\epsilon= \pm 1$ and a character $\mu$ of $S$, such that $\mu^{2}=1$, with the following properties.

(1) $\operatorname{tr} \tilde{\tau}(s z)=\epsilon \mu(s) \zeta(z)$, for $s \in S, s \neq 1$, and $z \in Z$.

(2) $p^{r}-\epsilon=k|S|$, for an integer $k$.

(3) $\operatorname{tr} \tilde{\tau} \mid S=k \mathrm{R}_{S}+\epsilon \mu$, where $\mathrm{R}_{S}$ is the character of the regular representation of $S$.

(4) The character $\mu$ is non-trivial if and only if $|S|$ is even and $k$ is odd.

Proof. This is a special case of [8] Theorem 13.32: to translate the notation, our $\tau$ is $\chi$ in [8], while $\tilde{\tau}$ is $\hat{\chi}$ and $\zeta$ is $\beta$. Otherwise, conventions are the same.

Remarks.

(1) The formulas in the proposition show that the character $\operatorname{tr} \tilde{\tau} \mid S$ of $S$ is determined by the group orders $|S|$ and $|V|$. Indeed, if $|S| \geqslant 3$, the invariants $k, \epsilon, \mu$ are individually determined by the group orders. When $|S|=2$, the character is determined but the invariants are not. In all cases, the character $\operatorname{tr} \tilde{\tau} \mid S$ depends only on the linear $\mathbb{F}_{p}$-representation of $S$ afforded by $V$.

(2) Let $S^{\prime}$ be a cyclic group, of order prime to $p$, equipped with a surjective homomorphism $S^{\prime} \rightarrow S$. One may inflate $\tilde{\tau}$ to a representation $\tilde{\tau}^{\prime}$ of $S^{\prime} \ltimes P$ and then use the proposition to write down $\operatorname{tr} \tilde{\tau}^{\prime} \mid S^{\prime}$.

Character computations of this sort feature in [1], especially (8.6.1) Theorem, and have been widely used. However, the account in [1] deals only with the case where the symplectic $\mathbb{F}_{p} S$-representation $P / Z$ is indecomposable. The 
proposition gives the exact formula for a wider class of cases. It neatly avoids an estimation process at a point where absolute precision is essential ( $c f .4 .4$, 4.5 below).

2.3. Suppose that the space $V$ of 2.1 decomposes as a direct sum of nonzero subspaces $V_{1}, V_{2}$, orthogonal with respect to the alternating form (2.1.2), say

$$
V=V_{1} \perp V_{2}
$$

Let $P_{i}$ be the inverse image of $V_{i}$ in $P$. The commutator group $\left[P_{1}, P_{2}\right]$ is trivial, that is, $P_{1}$ commutes with $P_{2}$. Moreover, each $P_{i}$ is H-cyclic with centre $Z$.

The obvious map $P_{1} \times P_{2} \rightarrow P$ is a surjective homomorphism with kernel $\left\{\left(z, z^{-1}\right): z \in Z\right\}$. That is, $P$ is the central product of its subgroups $P_{1}, P_{2}$. As in 2.1 Lemma, the group $P_{i}$ admits a unique irreducible representation $\tau_{i}$ containing the character $\zeta$ of $Z$. The representation $\tau_{1} \otimes \tau_{2}$ factors through the quotient map $\pi: P_{1} \times P_{2} \rightarrow P$ and so $\tau \circ \pi \cong \tau_{1} \otimes \tau_{2}$ : one may reasonably write

$$
\tau=\tau_{1} \otimes \tau_{2}
$$

2.4. Let $S$ be a cyclic group of automorphisms of $P$, as in 2.2 Definition, and suppose that the factors $V_{i}$ in $(2.3 .1)$ are $S$-invariant. It follows that the subgroups $P_{i}$ of $P$ are normalized by $S$. Let $S_{i}$ be the image of $S$ in Aut $P_{i}$. Following the procedure of 2.2 Lemma, we form the representation $\tilde{\tau}_{i}$ of $S_{i} \ltimes P_{i}$. We inflate $\tilde{\tau}_{i}$ to a representation $\tilde{\tau}_{i}^{S}$ of $S \ltimes P_{i}$. We can equally set $\tau=\tau_{1} \otimes \tau_{2}$ as in (2.3.2) and extend it to a representation $\tilde{\tau}$ of $S \ltimes P$ as before. We then have

$$
\operatorname{tr} \tilde{\tau}(s)=\operatorname{tr} \tilde{\tau}_{1}^{S}(s) \cdot \operatorname{tr} \tilde{\tau}_{2}^{S}(s), \quad s \in S
$$

\section{Conductor estimate For PRIMitive Representations}

We give a lower bound, in terms of ramification structure, for the Swan exponent of a certain class of representations of the Weil group. Before we start, we lay down some notation and conventions to remain in force for the rest of the paper.

\section{Notation and conventions.}

(1) Let $\mathcal{W}_{F}$ be the Weil group of a chosen separable closure $\bar{F} / F$. When speaking of a "representation of $\mathcal{W}_{F}$ " we mean a "smooth complex representation of $\mathcal{W}_{F}$ ". Let $\mathcal{J}_{F}$ be the inertia subgroup of $\mathcal{W}_{F}$ and $\mathcal{P}_{F}$ the wild inertia subgroup. 
(2) Let $\mathfrak{p}_{F}$ be the maximal ideal of the discrete valuation ring in $F$. If $k \geqslant 1$ is an integer, then $U_{F}^{k}$ is the unit group $1+\mathfrak{p}_{F}^{k}$. The residue field of $F$ is $\mathbb{k}_{F}$.

(3) We use the conventions of [12] when dealing with ramification groups, their numberings and the Herbrand functions $\varphi, \psi$.

3.1. An irreducible representation $\sigma$ of $\mathcal{W}_{F}$ is called primitive if $\operatorname{dim} \sigma>1$ and if $\sigma$ is not induced from a representation of $\mathcal{W}_{K}$, where $K / F$ is a finite field extension with $K \neq F$.

Hypothesis. For the rest of this section, we suppose that the representation $\sigma$ is primitive.

The restriction $\sigma \mid \mathcal{P}_{F}$ is then irreducible and the finite $p$-group $\sigma\left(\mathcal{P}_{F}\right)$ is $\mathrm{H}$ cyclic [11], Theorem 1. Consequently, $\operatorname{dim} \sigma=p^{r}$, for some $r \geqslant 1$. Let $\bar{\sigma}$ be the projective representation defined by $\sigma$ and set $\mathcal{W}_{K}=\operatorname{Ker} \bar{\sigma}$. In particular, $\sigma\left(\mathcal{W}_{K}\right)$ is the centre of $\sigma\left(\mathcal{W}_{F}\right)$, so $\sigma \mid \mathcal{W}_{K}$ is a multiple of a character $\zeta_{\sigma}$ of $\mathcal{W}_{K}$.

Let $T / F$ be the maximal tamely ramified sub-extension of $K / F$. The group $\Delta=\operatorname{Gal}(K / T)$ is elementary abelian of order $p^{2 r}$. Since $\sigma\left(\mathcal{P}_{F}\right)$ is H-cyclic, the pairing

$$
(x, y) \longmapsto \zeta_{\sigma}\left(x y x^{-1} y^{-1}\right), \quad x, y \in \mathcal{W}_{T},
$$

induces a bilinear form

$$
h_{\sigma}: \Delta \times \Delta \longrightarrow \mathbb{F}_{p}
$$

that is alternating and nondegenerate. The natural action of $\Theta=\operatorname{Gal}(T / F)$ on $\Delta$ fixes $h_{\sigma}$, so $\left(\Delta, h_{\sigma}\right)$ provides a symplectic representation of $\Theta$ over the field $\mathbb{F}_{p}$. A crucial point is the following [9] Theorem 4.1.

Proposition. The symplectic $\mathbb{F}_{p}$-representation of $\Theta$ on $\Delta$ is $\Theta$-anisotropic, in that $\Delta$ has no non-zero $\Theta$-subspace on which $h_{\sigma}$ is identically zero.

It is usually convenient to impose a further normalization.

Lemma. There is a tamely ramified character $\chi$ of $\mathcal{W}_{F}$, such that the representation $\sigma^{\prime}=\chi \otimes \sigma$ has the following properties.

(1) The kernel of $\sigma^{\prime}$ is of the form $\mathcal{W}_{E}$, where $E / K$ is finite, cyclic and totally wildly ramified.

(2) The order of the character $\zeta_{\sigma^{\prime}}$ is finite and a power of $p$, with $\mathcal{W}_{E}=$ $\operatorname{Ker} \zeta_{\sigma^{\prime}}$. 
Proof. We construct the character $\chi$ in stages. First, there is an unramified character $\chi_{1}$ of $\mathcal{W}_{F}$ such that the representation $\sigma_{1}=\chi_{1} \otimes \sigma$ has finite image. The character $\operatorname{det} \sigma_{1}$ therefore has finite order. There exists a character $\chi_{2}$ of $\mathcal{W}_{F}$, of finite order relatively prime to $p$, such that $\chi_{2}^{p^{r}} \operatorname{det} \sigma_{1}$ has finite $p$-power order. In particular, $\chi_{2}$ is tamely ramified. Set $\sigma_{2}=\chi_{2} \otimes \sigma_{1}$, so that $\operatorname{det} \sigma_{2}$ has finite $p$-power order. The restriction of $\sigma_{2}$ to $\mathcal{W}_{K}$ is a multiple of the character $\zeta_{2}=\zeta_{\sigma} \cdot \chi_{2} \chi_{1} \mid \mathcal{W}_{K}$. By construction, $\zeta_{2}$ has finite $p$-power order.

Let $\operatorname{Ker} \zeta_{2}=\mathcal{W}_{E_{2}}$. Thus $E_{2} / K$ is a finite, cyclic $p$-extension. Viewing $\zeta_{2}$ as a character of $K^{\times}$via class field theory, the extension $E_{2} / K$ is totally ramified if and only if $\zeta_{2}\left(K^{\times}\right)=\zeta_{2}\left(U_{K}\right)$ or, equivalently, there is a Frobenius element $\phi$ of $\mathcal{W}_{K}$ such that $\zeta_{2}(\phi)=1$. So, suppose we have a Frobenius $\phi$ for which $\zeta_{2}(\phi) \neq 1$. There is an unramified character $\psi$ of $\mathcal{W}_{K}$, of finite, p-power order, such that $\psi \zeta_{2}(\phi)=1$. This character $\psi$ is the restriction of an unramified character $\chi_{3}$ of $\mathcal{W}_{F}$ of finite, $p$-power order. Write $\zeta_{3}=\chi_{3} \zeta_{2}$ and $\mathcal{W}_{E}=\operatorname{Ker} \zeta_{3}$. The extension $E / K$ is cyclic and totally ramified of $p$-power degree. Moreover, $\mathcal{W}_{E}=\operatorname{Ker} \sigma_{3}$, where $\sigma_{3}=\chi_{3} \otimes \sigma_{2}$, and all assertions have been proved for $\sigma^{\prime}=\sigma_{3}$.

Remark. Replacing $\sigma$ by $\sigma^{\prime}$ has no effect on the pairing $h_{\sigma}$ or the fields $K, T$. The Tame Multiplicity Theorem holds for $\sigma$ if and only if it holds for $\sigma^{\prime}$.

3.2. In the notation of 3.1 , we analyze the symplectic $\mathbb{F}_{p}$-representation of $\Theta$ provided by $\Delta$. Let $J_{K / T}$ be the set of ramification jumps of $K / T$, in the upper numbering. Since $K / T$ is abelian, these jumps are positive integers, by the Hasse-Arf Theorem [12] V Théorème 1. Observe that, for a real number $x \geqslant 0$, the ramification group $\Delta^{x}$ is an $\mathbb{F}_{p} \Theta$-subspace of $\Delta$.

Proposition. Let $j \in J_{K / T}$.

(1) The restriction of $h_{\sigma}$ to $\Delta^{j}$ is nondegenerate.

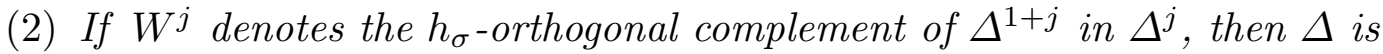
the orthogonal sum of the spaces $W^{j}, j \in J_{K / T}$.

Proof. If $X$ is a subspace of $\Delta$, let $X^{\perp}$ be its $h_{\sigma^{-}}$-orthogonal complement in $\Delta$. For an integer $j \geqslant 1$, the radical of the alternating form $h_{\sigma} \mid \Delta^{j} \times \Delta^{j}$ is $\Delta^{j} \cap\left(\Delta^{j}\right)^{\perp}$. This is an $\mathbb{F}_{p} \Theta$-subspace of $\Delta$ on which $h_{\sigma}$ is null. Since $h_{\sigma}$ is $\Theta$-anisotropic, $\Delta^{j} \cap\left(\Delta^{j}\right)^{\perp}=0$ whence (1) follows.

If $j \in J_{K / T}$, then $\Delta^{1+j}$ is trivial or equal to $\Delta^{j^{\prime}}$, where $j^{\prime}$ is the least element of $J_{K / T}$ strictly greater than $j$. Assertion (2) now follows from (1). 
3.3. We continue with the notation of $3.1,3.2$ to establish a lower bound on the Swan exponent $\operatorname{sw}(\sigma)$.

First, we specify a family of Lagrangian subspaces of the alternating space $\left(\Delta, h_{\sigma}\right)$. For each $j \in J_{K / T}$, let $\Xi(j)$ be a Lagrangian subspace of the nondegenerate space $W^{j}$. The various $\Xi(j)$ are mutually orthogonal, and so $\Xi=\sum_{j} \Xi(j)$ is Lagrangian. A Lagrangian subspace of this form will be called J-split.

Theorem. Let $\Xi$ be a J-split Lagrangian subspace of $\Delta$. If $K^{\Xi}=L$, then $J_{L / T}=J_{K / T}$. If $j_{\infty}$ is the largest element of $J_{L / T}$ and $e(T \mid F)=e$ then

$$
e \operatorname{sw}(\sigma) \geqslant \psi_{L / T}\left(j_{\infty}\right)+p^{r} j_{\infty} \geqslant\left(1+p^{r}\right) j_{\infty}
$$

Proof. We may assume, without loss, that the representation $\sigma$ has been normalized as in 3.1 Lemma. In particular, $\operatorname{Ker} \sigma=\mathcal{W}_{E}$, where $E / K$ is cyclic and totally wildly ramified. The extension $E / F$ is Galois.

By construction, the extensions $K / T$ and $L / T$ have the same jumps, $J_{L / T}=$ $J_{K / T}$. Let $\widetilde{\Delta}=\operatorname{Gal}(E / T), \widetilde{\Xi}=\operatorname{Gal}(E / L)$. Since $\Xi$ is a Lagrangian subspace of $\Delta$, the extension $E / L$ is abelian and totally wildly ramified. The Artin Reciprocity isomorphism therefore induces a surjective homomorphism

$$
a_{L}: U_{L}^{1} \longrightarrow \widetilde{\Xi}=\operatorname{Gal}(E / L) .
$$

Let $x \in \Delta^{j_{\infty}} \cap \Xi$ be non-trivial, and choose $y \in \Delta^{j_{\infty}}$ such that $h_{\sigma}(x, y) \neq$ 0 . We have $\Delta^{j_{\infty}}=\Delta_{k_{\infty}}$, where $k_{\infty}=\psi_{K / T}\left(j_{\infty}\right)$, and so $x$ is an element of $\Delta_{k_{\infty}} \cap \Xi=\Xi_{k_{\infty}}$. However,

$$
\Xi_{k_{\infty}}=\Xi^{\varphi_{K / L}\left(k_{\infty}\right)}=\Xi^{\psi_{L / T}\left(j_{\infty}\right)}
$$

as follows from the transitivity relation $\psi_{K / T}=\psi_{K / L} \circ \psi_{L / T}$. Choose an inverse image $\tilde{x}$ of $x$ in $\widetilde{\Xi}^{\psi_{L / T}\left(j_{\infty}\right)}$. As Galois operator on $E$ therefore, we have $\tilde{x}=$ $a_{L}(v)$, for some $\psi_{L / T}\left(j_{\infty}\right)$-unit $v$ of $L$ (by the higher ramification theorem of local class field theory [12] XV Théorème 1 Corollaire 3).

On the other hand, $y$ acts on $L$ as an element of

$$
(\widetilde{\Delta} / \widetilde{\Xi})^{j_{\infty}}=(\Delta / \Xi)^{j_{\infty}}=(\Delta / \Xi)_{\psi_{L / T}\left(j_{\infty}\right)}
$$

The definition of the lower ramification sequence implies that, if $z \in U_{L}^{k}$, for some $k \geqslant 1$, then $z^{y} / z$ is a $\left(k+\psi_{L / T}\left(j_{\infty}\right)\right)$-unit of $L$. 
Choose an inverse image $\tilde{y}$ of $y$ in $\widetilde{\Delta}^{j}$. Therefore

$$
\tilde{y}^{-1} \tilde{x} \tilde{y} \tilde{x}^{-1}=a_{L}\left(v^{\tilde{y}} v^{-1}\right)=a_{L}(u)
$$

where $u=v^{\tilde{y}} v^{-1}=v^{y} v^{-1}$ is a $2 \psi_{L / T}\left(j_{\infty}\right)$-unit of $L$.

Set $\sigma \mid \mathcal{W}_{T}=\tau$. The representation $\tau$ is irreducible. Since $\Xi$ is Lagrangian, $\tau$ is induced from a character $\phi$ of $\mathcal{W}_{L}$ extending the character $\zeta_{\sigma}$ of $\mathcal{W}_{K}(2.1$ Lemma). By construction, $\zeta_{\sigma}\left[y^{-1}, x\right]=\zeta_{\sigma}\left[\tilde{y}^{-1}, \tilde{x}\right] \neq 1$. So, if we view $\phi$ as a character of $L^{\times}$via class field theory, it is non-trivial on $2 \psi_{L / T}\left(j_{\infty}\right)$-units of $L$. That is, $\operatorname{sw}(\phi) \geqslant 2 \psi_{L / T}\left(j_{\infty}\right)$. Let $w_{L / T}$ be the wild exponent of the extension $L / T$ ( $c f$. (5.1.1) below). The standard induction formula reads

$$
\begin{aligned}
\operatorname{sw}(\tau) & =\operatorname{sw}(\phi)+w_{L / T} \\
& \geqslant 2 \psi_{L / T}\left(j_{\infty}\right)+w_{L / T} .
\end{aligned}
$$

Since $[L: T]=p^{r}$ and $j_{\infty}$ is the largest jump of $L / T$, we have

$$
\psi_{L / T}\left(j_{\infty}\right)=p^{r} j_{\infty}-w_{L / T}
$$

by [4] 1.6 Proposition. It follows that $\operatorname{sw}(\tau) \geqslant \psi_{L / T}\left(j_{\infty}\right)+p^{r} j_{\infty}$. The Herbrand function satisfies $\psi_{L / T}(x) \geqslant x$, for all $x \geqslant 0$, so we further have

$$
\mathrm{sw}(\tau) \geqslant \psi_{L / T}\left(j_{\infty}\right)+p^{r} j_{\infty} \geqslant\left(1+p^{r}\right) j_{\infty}
$$

Since $\operatorname{sw}(\tau)=e \operatorname{sw}(\sigma)$, we are done.

3.4. The theorem of 3.3, and its proof, apply unchanged in greater generality. We shall not use the fact here, but this is a convenient place to record it. Suppose only that the irreducible representation $\sigma$ is H-cyclic, in the sense of [4]: this means that $\sigma \mid \mathcal{P}_{F}$ is irreducible and that the finite $p$-group $\sigma\left(\mathcal{P}_{F}\right)$ is H-cyclic in the sense of 2.1. We can use all the same notation relative to $\sigma$. The inequalities (3.3.1) then hold, provided the alternating form $h_{\sigma}$ is nondegenerate on $\Delta^{j}$.

\section{Certain primitive Representations}

In this section, we prove the Tame Multiplicity Theorem for a certain class of primitive representations of $\mathcal{W}_{F}$.

4.1. Let $\sigma$ be a primitive irreducible representation of $\mathcal{W}_{F}$. Say that $\sigma$ is called absolutely ramified if the associated projective representation $\bar{\sigma}$ factors through a finite Galois group $\operatorname{Gal}(L / F)$ for which $L / F$ is totally ramified. 
Theorem. If $\sigma$ is an irreducible, primitive, absolutely ramified representation of $\mathcal{W}_{F}$, then $m(\sigma) \leqslant \operatorname{sw}(\sigma)$.

The proof will occupy the rest of the section.

4.2. We normalize $\sigma$ as permitted by (3.1) Lemma and use the notation developed in 3.1. Thus $\operatorname{Ker} \bar{\sigma}=\mathcal{W}_{K}$, where $K / F$ is totally ramified. Let $T / F$ be the maximal tame sub-extension of $K / F$. In addition, $\operatorname{Ker} \sigma=\mathcal{W}_{E}$ where $E / K$ is cyclic and totally wildly ramified.

Set $\Gamma=\operatorname{Gal}(K / F), \Delta=\operatorname{Gal}(K / T)$ and $\Theta=\operatorname{Gal}(T / F)$. Therefore $\Delta$ is elementary abelian of order $p^{2 r}=(\operatorname{dim} \sigma)^{2}$ and $\Theta$ is cyclic of order prime to $p$. The restriction of $\sigma$ to $\mathcal{W}_{K}$ is a multiple of a character $\zeta_{\sigma}$ and the group $\widetilde{\Delta}=\operatorname{Gal}(E / T)$ is an H-cyclic $p$-group with centre $\operatorname{Gal}(E / K)$. The subgroup $\Delta$ admits a complement $\Sigma$ in $\Gamma$. Thus $\Sigma \cap \Delta=\{1\}$ and $\Gamma=\Sigma \Delta$. Restriction of operators induces an isomorphism $\Sigma \cong \Theta$. In particular, $\Sigma$ is cyclic of order $e=e(T \mid F)$.

Let $h_{\sigma}$ be the commutator pairing as in (3.1.1). The pair $\left(\Delta, h_{\sigma}\right)$ affords an anisotropic, symplectic $\mathbb{F}_{p}$-representation of $\Sigma$, of dimension $2 r$. We review the classification of such representations, following [1].

Choose an algebraic closure $\overline{\mathbb{F}}_{p} / \mathbb{F}_{p}$, and write $\operatorname{Gal}\left(\overline{\mathbb{F}}_{p} / \mathbb{F}_{p}\right)=\Omega$. Let $\chi: \Sigma \rightarrow$ $\overline{\mathbb{F}}_{p}^{\times}$be a homomorphism and let $\mathbb{F}_{p}(\chi)$ be the field generated by the values $\chi(s)$, $s \in \Sigma$. The group $\Sigma$ acts on $\mathbb{F}_{p}(\chi)$ via the character $\chi$, that is,

$$
s: x \longmapsto \chi(s) x, \quad s \in \Sigma, x \in \mathbb{F}_{p}(\chi) .
$$

The group $\Omega$ acts on $\operatorname{Hom}\left(\Sigma, \overline{\mathbb{F}}_{p}\right)$ in a natural way. The map $\chi \mapsto \mathbb{F}_{p}(\chi)$ then induces a bijection between $\Omega \backslash \operatorname{Hom}\left(\Sigma, \overline{\mathbb{F}}_{p}\right)$ and the set of isomorphism classes of irreducible $\mathbb{F}_{p}$-representations of $\Sigma$.

\section{Proposition.}

(1) For $\chi \in \operatorname{Hom}\left(\Sigma, \overline{\mathbb{F}}_{p}^{\times}\right)$, the following conditions are equivalent.

(a) The representation $\mathbb{F}_{p}(\chi)$ is symplectic, that is, it admits a nondegenerate, $\Sigma$-invariant, alternating form.

(b) The character $\chi^{-1}$ is $\Omega$-conjugate, but not equal, to $\chi$.

(c) The field $\mathbb{F}_{p}(\chi)$ satisfies $\left[\mathbb{F}_{p}(\chi): \mathbb{F}_{p}\right]=p^{2 d}$, for an integer $d \geqslant 1$, and $\chi(\Sigma)$ is contained in the subgroup of $\mathbb{F}_{p}(\chi)^{\times}$of order $1+p^{d}$.

(2) Suppose that $\mathbb{F}_{p}(\chi)$ is symplectic. Any nonzero $\Sigma$-invariant alternating form on $\mathbb{F}_{p}(\chi)$ is $\Sigma$-anisotropic. Any two such forms are $\Sigma$-isometric. 
(3) A finite $\mathbb{F}_{p}$-representation $U$ of $\Sigma$ provides a symplectic anisotropic representation of $\Sigma$ if and only if there exist $\chi_{j} \in \operatorname{Hom}\left(\Sigma, \overline{\mathbb{F}}_{p}^{\times}\right), 1 \leqslant j \leqslant r$, such that

(a) each $\mathbb{F}_{p}\left(\chi_{j}\right)$ is symplectic;

(b) if $i \neq j$, then $\chi_{i}$ is not $\Omega$-conjugate to $\chi_{j}$;

(c) $U=\bigoplus_{1 \leqslant j \leqslant r} \mathbb{F}_{p}\left(\chi_{j}\right)$.

The proposition is taken from section 8.2 of [1]. It may equally be viewed as an instance of the more general classification in [9] Theorem 8.1, although some effort of translation would be required.

Remark. If $\chi$ has order $a$ and satisfies the conditions in part (1) of the proposition, then

(a) $a \geqslant 3$ and

(b) the integer $d$ is the least for which $1+p^{d}$ is divisible by $a$.

4.3. In the same situation, we analyze the symplectic $\mathbb{F}_{p}$-representation of $\Sigma$ on $\Delta=\operatorname{Gal}(K / T)$. Following 4.2 Proposition (2), it is only the structure of the linear $\mathbb{F}_{p} \Sigma$-representation $\Delta$ that need concern us.

Recall that $J_{K / T}$ is the set of (upper) ramification jumps of $K / T$. For $j \in$ $J_{K / T}$, define $W^{j}$ as in 3.2 Proposition.

Proposition. For all $j \in J_{K / T}$, the $\mathbb{F}_{p} \Sigma$-space $W^{j}$ is irreducible.

Proof. Let $k \in \mathbb{Z}, k \geqslant 1$. The group $\Delta^{k}$ is the image of the unit group $U_{T}^{k}$ under the Artin reciprocity map $T^{\times} \rightarrow \Delta=\operatorname{Gal}(K / T)$. This map is $\Sigma$-equivariant and $W^{j}, j \in J_{K / T}$, is so realized as a $\Sigma$-quotient of $U_{T}^{j} / U_{T}^{1+j}$.

The natural action of $\Sigma=\operatorname{Gal}(T / F)$ on $\mathfrak{p}_{T} / \mathfrak{p}_{T}^{2}$ is given by a faithful character $\theta: \Sigma \rightarrow \mathbb{k}_{F}^{\times}$. The natural action on $\mathfrak{p}_{T}^{j} / \mathfrak{p}_{T}^{1+j}, j \geqslant 1$, is therefore implemented by $\theta^{j}$. The character $\theta^{j}$ induces an algebra homomorphism $\mathbb{F}_{p} \Sigma \rightarrow \mathbb{k}_{F}$, the image of which is necessarily a subfield of $\mathbb{k}_{F}$. The $\mathbb{F}_{p} \Sigma$-module $U_{T}^{j} / U_{T}^{1+j} \cong \mathfrak{p}_{T}^{j} / \mathfrak{p}_{T}^{1+j}$ is therefore isotypic. However, $W^{j}$ is anisotropic, so 4.2 Proposition (3) implies that $W^{j}$ is a direct sum of mutually inequivalent irreducible $\mathbb{F}_{p} \Sigma$-modules. It is therefore irreducible, as required.

We underline some points made in the preceding proof. 
Corollary. Let $j \in J_{K / T}$.

(1) The symplectic $\mathbb{F}_{p}$-representation $W^{j}$ of $\Sigma$ is equivalent to $\mathbb{F}_{p}\left(\theta^{j}\right)$.

(2) Let $e=e(T \mid F)=|\Sigma|$. An element $s \in \Sigma$ has a non-trivial fixed point in $W^{j}$ if and only if $s^{\operatorname{gcd}(e, j)}=1$.

4.4. Twisting $\sigma$ with a character of $\operatorname{Gal}(T / F)$ has no effect on the assertion to be proved. We therefore assume that the character $\operatorname{det} \sigma$ is trivial on $\Sigma$ : this puts us in the situation of 2.2 Proposition.

The orthogonal decomposition $\Delta=\sum_{j \in J_{K / T}} W^{j}$ implies a canonical realization of the $\mathbb{F}_{p} \Sigma$-module $W^{j}$ as a subspace of $\Delta$ : let $\widetilde{W}^{j}$ be its inverse image in $\widetilde{\Delta}$. The construction outlined in 2.3,2.4 gives a representation $\sigma^{j}$ of $\Sigma \widetilde{W}^{j}$ and a tensor decomposition

$$
\sigma=\bigotimes_{j \in J_{K / T}} \sigma^{j}
$$

We may choose the factors $\sigma^{j}$ so that each character $\operatorname{det} \sigma^{j} \mid \Sigma$ is trivial. As in 4.3 Corollary (2), $\Sigma$ acts on $W^{j}$ via its quotient of order $e_{j}=e /(e, j)$, and that action is faithful.

Definition. Let $A$ be the set of positive divisors $a$ of $e$ of the form $e_{j}=e /(e, j)$, for some $j \in J_{K / T}$. For $a \in A$, set

$$
\sigma_{a}=\bigotimes_{\substack{j \in J_{K / T}, a=e_{j}}} \sigma^{j}
$$

Remark that $e=|\Sigma|$ is the lcm of the elements of $A$. Note also that a factor $\sigma_{a}$ may have several ramification jumps: this possibility is not excluded by 4.2 Proposition.

We work in the ring $\mathbb{Z} \widehat{\Sigma}$ of virtual characters of $\Sigma$. The elements of $\mathbb{Z} \widehat{\Sigma}$ are thus the formal linear combinations

$$
\boldsymbol{c}=\sum_{\chi \in \widehat{\Sigma}} c_{\chi} \chi
$$

in which the coefficients $c_{\chi}$ lie in $\mathbb{Z}$. Let $\mathbb{N} \widehat{\Sigma}$ be the "order" consisting of those $\boldsymbol{c} \in \mathbb{Z} \widehat{\Sigma}$ for which the coefficients $c_{\chi}$ are all non-negative. For $\boldsymbol{a}, \boldsymbol{b} \in \mathbb{Z} \widehat{\Sigma}$, we write $\boldsymbol{a} \geqslant \boldsymbol{b}$ when $\boldsymbol{a}-\boldsymbol{b} \in \mathbb{N} \widehat{\Sigma}$. We also use the relation $\geqslant$ to compare elements of $\mathbb{Q} \widehat{\Sigma}$ in the obvious way. 
Definition. Let $a \in A$.

(1) Let $q_{a}$ be the least power of $p$ such that $1+q_{a}$ is divisible by $a$ and define $\ell(a)$ as the number of $j \in J_{K / T}$ for which $a=e_{j}$.

(2) Define the positive integer $k_{a}$ by

$$
a k_{a}=\left(q_{a}^{\ell(a)}-(-1)^{\ell(a)}\right) .
$$

(3) Let $\mu_{a}$ denote the trivial character of $\Sigma$ if $a$ is odd or $k_{a}$ is even. Otherwise, let $\mu_{a} \in \widehat{\Sigma}$ have order 2 .

(4) Let $\boldsymbol{\rho}_{a} \in \mathbb{Z} \widehat{\Sigma}$ be the sum of characters $\phi$ of $\Sigma$ such that $\phi^{a}=1$, and define

$$
R_{a}=k_{a} \boldsymbol{\rho}_{a}+(-1)^{\ell(a)} \mu_{a} \in \mathbb{Z} \widehat{\Sigma}
$$

Proposition. If $a \in A$, then

$$
\sigma_{a} \mid \Sigma=R_{a}
$$

and, moreover,

$$
\sigma \mid \Sigma=\prod_{a \in A} R_{a}
$$

the product being taken in $\mathbb{Z} \widehat{\Sigma}$.

Proof. This follows directly from 2.2 Proposition and (2.4.1).

4.5. We treat a special case of 4.1 Theorem, working directly from (4.4.2).

Proposition. If the set $A$ has exactly one element, then $m(\sigma) \leqslant \operatorname{sw}(\sigma)$.

Proof. The lcm of the elements of $A$ is $e$, so $A=\{e\}$. Moreover, $q_{e}^{\ell(e)}=p^{r}=$ $\operatorname{dim} \sigma$.

Suppose first that $\ell(e)$ is odd, so that $m(\sigma)=k_{e}=\left(p^{r}+1\right) / e$. We have to show that $p^{r}+1 \leqslant e \operatorname{sw}(\sigma)$. By 3.3 Theorem, $e \operatorname{sw}(\sigma) \geqslant\left(p^{r}+1\right) j_{\infty}$, where $j_{\infty}$ is the largest element of $J_{K / T}$. As $j_{\infty}$ is a positive integer ( $c f .3 .2$ ), so

$$
e \operatorname{sw}(\sigma) \geqslant\left(p^{r}+1\right) j_{\infty} \geqslant\left(p^{r}+1\right)
$$


as required. Suppose, on the other hand, that $\ell(e)$ is even. In this case, $e$ divides $p^{r}-1$, so $e \leqslant p^{r}-1$ and

$$
e m(\sigma)=p^{r}-1+e \leqslant 2\left(p^{r}-1\right) .
$$

On the other hand, as $\ell(e)$ is even so $j_{\infty} \geqslant 2$. Therefore

$$
e m(\sigma) \leqslant 2\left(p^{r}-1\right)<\left(p^{r}+1\right) j_{\infty} \leqslant e \operatorname{sw}(\sigma) .
$$

This completes the proof.

We reflect briefly on the proof of this proposition.

Corollary of proof. In the situation of the proposition, if $m(\sigma)=\mathrm{sw}(\sigma)$ then $J_{K / T}=\{1\}$.

Proof. If $\ell(e)$ is even then, as we have just seen, $m(\sigma)<\operatorname{sw}(\sigma)$, so suppose $\ell(e)$ is odd. If $\ell(e) \neq 1$, then $j_{\infty} \geqslant 3$ and

$$
e m(\sigma)=1+p^{r}<\left(1+p^{r}\right) j_{\infty} \leqslant e \operatorname{sw}(\sigma) .
$$

So, we assume $\ell(e)=1$ and $J_{K / T}=\left\{j_{\infty}\right\}$. In this case, if $j_{\infty}>1$ then $m(\sigma)<\operatorname{sw}(\sigma)$.

4.6. Assume now that $A$ has at least two elements. We can make some simplifying approximations. The expressions (4.4.1), (4.4.2) imply

$$
\sigma_{a} \mid \Sigma=R_{a} \leqslant q_{a}^{\ell(a)} d_{a} \frac{\boldsymbol{\rho}_{a}}{a}
$$

where

$$
d_{a}= \begin{cases}1+q_{a}^{-\ell(a)} & \text { if } \ell(a) \text { is odd } \\ 1+(a-1) q_{a}^{-\ell(a)} & \text { if } \ell(a) \text { is even }\end{cases}
$$

If $a, b \in A$, then

$$
\frac{\boldsymbol{\rho}_{a}}{a} \frac{\boldsymbol{\rho}_{b}}{b}=\frac{\boldsymbol{\rho}_{c}}{c}
$$

where $c$ is the lcm of $a$ and $b$. So, taking the product over $a \in A$, we get

$$
\sigma \mid \Sigma \leqslant \operatorname{dim} \sigma \prod_{a \in A} d_{a} \frac{\boldsymbol{\rho}_{e}}{e}
$$

whence

$$
m(\sigma) \leqslant \frac{\operatorname{dim} \sigma}{e} \prod_{a \in A} d_{a}
$$

So, we are reduced to proving: 
Proposition. If $|A| \geqslant 2$, then

$$
\prod_{a \in A} d_{a} \leqslant \frac{e \operatorname{sw}(\sigma)}{\operatorname{dim} \sigma}
$$

Proof. Let $j_{\infty}$ be the largest element of $J_{K / T}$. Let $\Xi$ be a $J$-split Lagrangian subspace of the symplectic space $\Delta$ as in 3.3. Let $L$ be the fixed field of $\Xi$ and recall that $J_{K / T}$ is equal to the set $J_{L / T}$ of jumps of $L / T$.

Lemma 1. Suppose that $A$ has at least two elements. If $a \in A$, then $a \geqslant 3$ and $d_{a} \leqslant a /(a-1)$.

Proof. For the first assertion, see 4.2 Remark (a). For the second, suppose first that $\ell(a)$ is even, whence $d_{a}=1+(a-1) q_{a}^{-\ell(a)}$. By definition, $a$ divides $1+q_{a}$ whence $a-1 \leqslant q_{a}$. Therefore

$$
d_{a} \leqslant 1+q_{a}^{-1} \leqslant 1+(a-1)^{-1}=a /(a-1) .
$$

The case of $\ell(a)$ odd is similar but even easier.

It follows that

$$
\prod_{a \in A} d_{a} \leqslant \prod_{a \in A} \frac{a}{a-1}
$$

Let $t \geqslant 2$ be the number of elements of $A$. The largest element of $A$ is therefore, at least, $t+2$ and all elements are $\geqslant 3$. Consequently,

$$
\prod_{a \in A} d_{a} \leqslant \frac{3}{2} \frac{4}{3} \cdots \frac{t+2}{t+1} \leqslant \frac{t+2}{2} \leqslant t .
$$

Lemma 2. If $h$ denotes the number of jumps of $L / T$, then

$$
h<p^{-r} \psi_{L / T}\left(j_{\infty}\right)+j_{\infty} .
$$

Proof. The jumps of the abelian extension $L / T$ are positive integers, so $h \leqslant j_{\infty}$ and $h-j_{\infty} \leqslant 0<p^{-r} \psi_{L / T}\left(j_{\infty}\right)$, as required.

Assembling these relations and applying (3.3.1), we get

$$
\prod_{a \in A} d_{a} \leqslant t \leqslant h<e \operatorname{sw}(\sigma) / p^{r}
$$

as required to complete the proof of the proposition.

This finishes the proof of 4.1 Theorem. 


\section{An estimate of THE DifFERENT}

Preliminary to the proof of the general case of the main theorem, we make an estimate of the wild exponent $w_{K / F}$ of a class of finite extensions $K / F$. It is not remotely sharp (see 5.2 Example) but is adequate for our purposes.

5.1. Let $K / F$ be a finite separable extension, with $K \subset \bar{F}$. The wild exponent $w_{K / F}$ of $K / F$ is

$$
\begin{aligned}
w_{K / F} & =d_{K / F}+1-e(K \mid F) \\
& =\operatorname{sw}\left(\operatorname{Ind}_{K / F} 1_{K}\right),
\end{aligned}
$$

where $d_{K / F}$ is the exponent of the different of $K / F$ and $1_{K}$ denotes the trivial character of $\mathcal{W}_{K}$.

5.2. Let $E / F$ be a finite, totally ramified, Galois extension. $\operatorname{Set} \operatorname{Gal}(E / F)=\Gamma$ and let $\Delta$ be the wild inertia subgroup of $\Gamma$. As in 1.1, $\Delta$ is the unique $p$-Sylow subgroup of $\Gamma$ and admits a complement $\Sigma$ in $\Gamma$. In particular, $\Sigma$ is cyclic of order prime to $p$.

Proposition. Let $\Phi$ be a subgroup of $\Gamma$, such that the index $(\Gamma: \Phi)$ is a power of $p$. If $K$ is the fixed field $E^{\Phi}$ of $\Phi$ in $E$, then

$$
w_{K / F} \geqslant|\Phi \backslash \Gamma / \Sigma|-1 \text {. }
$$

Proof. Recall that any two choices of the complement $\Sigma$ are conjugate in $\Gamma$. The assertion is therefore independent of the choice of $\Sigma$.

If $\Phi=\Gamma$ there is nothing to prove, so we assume otherwise.

Lemma 1. Let $\Xi$ be a normal subgroup of $\Gamma$ such that $\Xi \subset \Phi$ and let $f: \Gamma \rightarrow$ $\Gamma / \Xi$ be the quotient map.

(1) The group $f(\Sigma)$ is a complement of $f(\Delta)$ in $\Gamma / \Xi$.

(2) The map $f$ induces a $\Sigma$-equivariant bijection $\Phi \backslash \Gamma \rightarrow f(\Phi) \backslash f(\Gamma)$, and hence a bijection $\Phi \backslash \Gamma / \Sigma \rightarrow f(\Phi) \backslash f(\Gamma) / f(\Sigma)$.

Proof. Straightforward.

Continue with $\Xi$ as in the lemma. If we replace $E$ by $E^{\Xi}$, the extension $K / F$ is unchanged. The effect of the lemma is to show that, if the proposition holds for the configuration $F \subset K \subset E^{\Xi}$, then it holds for $F \subset K \subset E$. We may choose $\Xi$ so that $E^{\Xi} / F$ is a normal closure of $K / F$. It is therefore enough to prove the proposition under the assumption that $E / F$ is a normal closure of $K / F$. We henceforward assume this to be the case. 
Lemma 2. Let $\Theta$ be the smallest non-trivial ramification subgroup of $\Gamma$. The group $\Theta$ is elementary abelian and central in $\Delta$. It is not contained in $\Phi$.

Proof. The first assertions are given by [12] IV Prop. 7 and Prop. 10. If $\Theta$ were contained in $\Phi$ then $E^{\Theta} / F$ would be a normal extension containing $K / F$ and such that $\left[E^{\Theta}: F\right]<[E: F]$. Since $E / F$ is a normal closure of $K / F$, this is impossible.

Suppose for the moment that $\Gamma=\Phi \Theta$ or, equivalently, that $\Delta=(\Phi \cap \Delta) \Theta$. As $\Theta$ is central in $\Delta$, so $\Phi \cap \Delta$ is normal in $\Delta$ and $\Delta / \Phi \cap \Delta$ is abelian. Let $1_{\Phi}$ denote the trivial character of $\Phi$, and similarly for other groups. The Mackey formula gives the relations

$$
\begin{aligned}
\operatorname{Ind}_{\Phi}^{\Gamma} 1_{\Phi} \mid \Delta & =\operatorname{Ind}_{\Phi \cap \Delta}^{\Delta} 1_{\Phi \cap \Delta}, \\
\operatorname{Ind}_{\Phi}^{\Gamma} 1_{\Phi} \mid \Theta & =\operatorname{Ind}_{\Phi \cap \Theta}^{\Theta} 1_{\Phi \cap \Theta} .
\end{aligned}
$$

The restriction (5.2.2) is the direct sum of all characters $\chi$ of $\Phi \cap \Theta \backslash \Theta$. Any such character $\chi$ extends uniquely to a character $\chi_{\Delta}$ of $\Delta$ trivial on $\Phi \cap \Delta$ : one puts $\chi_{\Delta}(h r)=\chi(r), h \in \Phi \cap \Delta, r \in \Theta$. Consequently,

$$
\operatorname{Ind}_{\Phi \cap \Delta}^{\Delta} 1_{\Phi \cap \Delta}=\sum_{\chi \in(\Phi \cap \Theta \backslash \Theta)^{\wedge}} \chi_{\Delta} .
$$

If $\Gamma_{\chi}$ denotes the $\Gamma$-centralizer of $\chi_{\Delta} \in(\Delta / \Phi \cap \Delta)^{\wedge}$, then $\Gamma_{\chi}=\Sigma_{\chi} \Delta$, where $\Sigma_{\chi}$ is the $\Sigma$-centralizer of $\chi_{\Delta}$ (or, equivalently, of $\chi$ ). Consequently, there is a unique character $\chi_{\Sigma}$ of $\Gamma_{\chi}$ that extends $\chi_{\Delta}$ and is trivial on $\Sigma_{\chi}$. Therefore

$$
\operatorname{Ind}_{\Phi}^{\Gamma} 1_{\Phi}=\sum_{\chi \in \Sigma \backslash(\Phi \cap \Theta \backslash \Theta)^{\wedge}} \sum_{\eta \in\left(\Gamma_{\chi} / \Delta\right)^{\wedge}} \operatorname{Ind}_{\Gamma_{\chi}}^{\Gamma} \eta \chi_{\Sigma} .
$$

We calculate the contribution of each term here to the exponent $\operatorname{sw}\left(\operatorname{Ind}_{\Phi}^{\Gamma} 1_{\Phi}\right)=$ $w_{K / F}$.

If $\chi$ is trivial, then $\Gamma_{\chi}=\Gamma$ and we get a contribution of 0 . Otherwise, $\operatorname{Ind}_{\Gamma_{\chi}}^{\Gamma} \eta \chi_{\Sigma}$ has Swan exponent at least 1 , whence

$$
w_{K / F} \geqslant \sum_{\substack{\chi \in \Sigma \backslash(\Phi \cap \Theta \backslash \Theta)^{\wedge} \\ \chi \neq 1}}\left(\Gamma_{\chi}: \Delta\right) .
$$

However, $\left|\Sigma \backslash(\Phi \cap \Theta \backslash \Theta)^{\wedge}\right|=|\Phi \backslash \Gamma / \Sigma|$, and so $w_{K / F} \geqslant|\Phi \backslash \Gamma / \Sigma|-1$ in this case. 
Example. Remark here that, once the trivial character $\chi$ is excluded, all groups $\Gamma_{\chi}$ are the same: they depend only on the denominator of $j$, where $\Theta=\Gamma^{j} \neq$ $\Gamma^{j+\epsilon}, \epsilon>0$. All characters $\eta \chi_{\Sigma}$ have the same slope, namely $j$. The index $\left(\Gamma: \Gamma_{\chi}\right)$, for $\chi \neq 1$, is the g.c.d. of $|\Sigma|$ and the denominator of $j$. So, for $\chi \neq 1$, the inner sum has Swan exponent $j\left(\Gamma: \Gamma_{\chi}\right)\left(\Gamma_{\chi}: \Delta\right)=j|\Sigma|$. Therefore

$$
\operatorname{sw}\left(\operatorname{Ind}_{\Phi}^{\Gamma} 1_{\Phi}\right)=w_{K / F}=j|\Sigma|(|\Phi \backslash \Gamma / \Sigma|-1) .
$$

We return to the proof of 5.2 Proposition, assuming now that $\Phi \Theta \neq \Gamma$. Since the index $(\Gamma: \Phi)$ is a power of $p$, the group $\Phi$ contains a conjugate of $\Sigma$. Following the remark at the beginning of the proof, we may assume that $\Sigma \subset \Phi$.

Let $L=E^{\Phi \Theta}$. The first case above gives

$$
w_{K / L} \geqslant|\Phi \backslash \Phi \Theta / \Sigma|-1
$$

By induction on $[K: F]=(\Gamma: \Phi)$, we likewise have

$$
w_{L / F} \geqslant|f(\Phi) \backslash f(\Gamma) / f(\Sigma)|-1
$$

where $f: \Gamma \rightarrow \Gamma / \Theta$ is the quotient map. On the other hand,

$$
w_{K / F}=w_{K / L}+[K: L] w_{L / F}
$$

SO

$$
w_{K / F} \geqslant|\Phi \backslash \Phi \Theta / \Sigma|-1+[K: L](|f(\Phi) \backslash f(\Gamma) / f(\Sigma)|-1) .
$$

Under the canonical surjection $\bar{f}: \Phi \backslash \Gamma / S \rightarrow f(\Phi) \backslash f(\Gamma) / f(\Sigma)$ induced by the quotient map $f: \Gamma \rightarrow \Gamma / \Theta$, the fibre of the trivial coset $f(\Phi)=f(\Phi) f(\Sigma)$ is precisely $\Phi \backslash \Phi \Theta / \Sigma$. On the other hand, let $x=f(g) \notin f(\Phi)$. The fibre, under $\bar{f}$, of $f(\Phi) x f(\Sigma)$ is contained in $\Phi g \Sigma$. This comprises at most $[K: L]$ double cosets $\Phi g \Sigma$, whence the result follows.

\section{Proof of THE MAIN THEOREM}

We prove the Tame Multiplicity Theorem in the general case. Let $\sigma$ be an irreducible representation of $\mathcal{W}_{F}$ that is not tamely ramified: see 1.1 Remark (1). Since the assertion of the theorem is unaffected by tensoring $\sigma$ with an unramified character of $\mathcal{W}_{F}$, we may treat $\sigma$ as a representation of $\Gamma=\operatorname{Gal}(E / F)$, where $E / F$ is finite. Let $\Gamma_{0}, \Gamma_{1}$ be respectively the inertia and the wild inertia subgroups of $\Gamma$, and similarly for other finite Galois groups. 
6.1. Let $\sigma$ be an irreducible representation of $\Gamma=\operatorname{Gal}(E / F)$, with $\operatorname{sw}(\sigma)>0$. Let $\Sigma$ be a complement of $\Gamma_{1}$ in $\Gamma_{0}$.

Proposition. If $\sigma$ is absolutely ramified, that is, if $E / F$ is totally ramified, then $m(\sigma) \leqslant \operatorname{sw}(\sigma)$.

Proof. If $\sigma$ is primitive or of dimension one, the result holds by 4.1 Theorem or 1.1 Remark (2) respectively. We therefore suppose otherwise: there is a proper subgroup $\Delta$ of $\Gamma$ and an irreducible representation $\tau$ of $\Delta$ such that $\sigma=\operatorname{Ind}_{\Delta}^{\Gamma} \tau$. The representation $\tau$ is absolutely ramified and, by induction on dimension, we may assume that $m(\tau) \leqslant \mathrm{sw}(\tau)$.

Suppose first that $\Delta$ may be chosen to contain $\Gamma_{1}$. Thus $\Gamma=\Gamma_{0}=\Sigma \Delta$, and $\Delta$ is a normal subgroup of $\Gamma$. The Mackey formula gives

$$
\sigma\left|\Sigma=\operatorname{Ind}_{\Sigma \cap \Delta}^{\Sigma} \tau\right| \Sigma \cap \Delta=\tau \mid \Sigma
$$

whence $m(\sigma)=m(\tau)$. As $E^{\Delta} / F$ is tamely ramified, so $\operatorname{sw}(\sigma)=\operatorname{sw}(\tau)$ and we are done in this case.

We therefore assume that $\sigma$ cannot be induced from a proper subgroup of $\Gamma=\Gamma_{0}$ that contains $\Gamma_{1}$. Since $\Gamma / \Gamma_{1}$ is cyclic, the restriction $\sigma \mid \Gamma_{1}$ is irreducible. In particular, $\operatorname{dim} \sigma$ is a power of $p$. It follows that, if $\sigma$ is induced from a representation $\tau$ of a proper subgroup $\Delta$ of $\Gamma$, then $(\Gamma: \Delta)$ is a power of $p$ and, if $K=E^{\Delta}$, the extension $K / F$ is totally wildly ramified. We have $m(\tau) \leqslant \operatorname{sw}(\tau)$, and

$$
\operatorname{sw}(\sigma)=\operatorname{sw}(\tau)+w_{K / F} \operatorname{dim} \tau .
$$

We apply 5.2 Proposition. We adjust our choice of $\Sigma$, via conjugation by an element of $\Gamma_{1}$, to achieve $\Sigma \subset \Delta$. Let $\chi$ be a character of $\Sigma$. In the Mackey expansion

$$
\sigma \mid \Sigma=\sum_{g \in \Delta \backslash \Gamma / \Sigma} \operatorname{Ind}_{g^{-1} \Delta g \cap \Sigma}^{\Sigma}\left(\tau^{g} \mid g^{-1} \Delta g \cap \Sigma\right) .
$$

the trivial double coset gives the term $\tau \mid \Sigma$, in which $\chi$ occurs with multiplicity at most $m(\tau)$. The contribution from a non-trivial double coset contains $\chi$ with multiplicity at most $\operatorname{dim} \tau$ so, overall,

$$
m(\sigma) \leqslant m(\tau)+(|\Delta \backslash \Gamma / \Sigma|-1) \operatorname{dim} \tau .
$$

Comparing (6.1.1) with (6.1.2), the proposition of 5.2 implies

$$
m(\sigma) \leqslant \operatorname{sw}(\tau)+w_{K / F} \operatorname{dim} \tau=\operatorname{sw}(\sigma),
$$

as required.

We return to a more general situation. 
Corollary. Let $E / F$ be a finite Galois extension and let $\sigma$ be an irreducible representation of $\Gamma=\operatorname{Gal}(E / F)$, with $\operatorname{sw}(\sigma)>0$. If $\sigma \mid \Gamma_{0}$ is irreducible, then $m(\sigma) \leqslant \operatorname{sw}(\sigma)$.

Proof. The representation $\sigma_{0}=\sigma \mid \Gamma_{0}$ is irreducible and absolutely ramified. The proposition gives $m\left(\sigma_{0}\right) \leqslant \operatorname{sw}\left(\sigma_{0}\right)$. However, since $\Sigma \subset \Gamma_{0}$, we have $m\left(\sigma_{0}\right)=$ $m(\sigma)$. On the other hand, $\operatorname{sw}\left(\sigma_{0}\right)=\operatorname{sw}(\sigma)$, since $E^{\Gamma_{0}} / F$ is unramified.

Example. Example 2 of [3] 8.5 is interesting in this context. Suppose that $p=2$ and that $F$ contains a primitive cube root of unity. The construction in [3] yields a primitive representation $\sigma$ of dimension 8 , with $\operatorname{sw}(\sigma)=3$ and a unique ramification jump. (In the notation of 3.3 Theorem, this jump is $j_{\infty}$ and it has value 1.) If $\operatorname{Ker} \bar{\sigma}=\mathcal{W}_{K}$, and $T / F$ is the maximal tame sub-extension of $K / F$, then $[T: F]=9$ and $e(T \mid F)=3$. In particular, $\sigma$ is not absolutely ramified. If $T_{0} / F$ is the maximal unramified sub-extension of $T / F$, the restriction $\sigma \mid \mathcal{W}_{T_{0}}$ is irreducible but not primitive. A simple counting argument gives $m(\sigma)=3=$ $\operatorname{sw}(\sigma)$.

6.2. We complete the proof of the Tame Multiplicity Theorem. Let $\sigma$ be an irreducible representation of the finite group $\Gamma=\operatorname{Gal}(E / F)$ with $\operatorname{sw}(\sigma)>0$. Let $\Sigma$ be a complement of $\Gamma_{1}$ in $\Gamma_{0}$. If $\sigma \mid \Gamma_{0}$ is irreducible, the theorem is 6.1 Corollary. We therefore assume otherwise, so there exist a proper subgroup $\Delta$ of $\Gamma$ containing $\Gamma_{0}$ and an irreducible representation $\tau$ of $\Delta$ that induces $\sigma$. We choose $\Delta$ minimal with respect to this property, so that $\tau \mid \Delta_{0}$ is irreducible. By 6.1 Corollary, $m(\tau) \leqslant \mathrm{sw}(\tau)$ while

$$
\operatorname{sw}(\sigma)=(\Gamma: \Delta) \operatorname{sw}(\tau) .
$$

As $\Delta_{0}=\Gamma_{0}$ and $\Delta_{1}=\Gamma_{1}$, so $\Sigma$ is also a complement of $\Delta_{1}$ in $\Delta_{0}$. Applying the standard Mackey formula, we get

$$
\sigma \mid \Sigma=\sum_{g \in \Delta \backslash \Gamma / \Sigma} \operatorname{Ind}_{g^{-1} \Delta g \cap \Sigma}^{\Sigma}\left(\tau^{g} \mid g^{-1} \Delta g \cap \Sigma\right) .
$$

We have $\Gamma_{0}=\Sigma \Gamma_{1} \subset \Delta$, while any $\Gamma$-conjugate of $\Sigma$ is contained in $\Delta$. The canonical map $\Delta \backslash \Gamma \rightarrow \Delta \backslash \Gamma / \Sigma$ is therefore bijective. Consider the expression

$$
\sigma\left|\Sigma=\sum_{g \in \Delta \backslash \Gamma} \tau^{g}\right| \Sigma .
$$

If $\chi$ is a character of $\Sigma$, the multiplicity of $\chi$ in $\tau^{g}$ is that of $\chi^{g^{-1}}$ in $\tau$, whence at most $m(\tau)$. We conclude that $m(\sigma) \leqslant(\Gamma: \Delta) m(\tau)$. Since $m(\tau) \leqslant \operatorname{sw}(\tau)$, the desired relation $m(\sigma) \leqslant \mathrm{sw}(\sigma)$ follows from (6.2.1). 


\section{REFERENCES}

1. C.J. Bushnell and A. Fröhlich, Gauss sums and p-adic division algebras, Lecture Notes in Math., vol. 987, Springer, Berlin-Heidelberg New York, 1983.

2. C.J. Bushnell and G. Henniart, Langlands parameters for epipelagic representations of $\mathrm{GL}_{n}$, Math. Annalen 358 (2014), 433-463.

3. Higher ramification and the local Langlands correspondence, Annals of Mathematics (2) 185 (2017), no. 3, 919-955.

4. Local Langlands correspondence and ramification for Carayol representations, arXiv: 1611.09258v3. To appear in Compositio Math.

5. G. Glauberman, Correspondences of characters for relatively prime operator groups, Canadian J. Math. 20 (1968), 1465-1488.

6. D. Gorenstein, Finite groups, AMS Chelsea Publishing, Providence RI, 2012.

7. G. Henniart, Représentations du groupe de Weil d'un corps local, L'Ens. Math. Sér. II 26 (1980), 155-172.

8. I.M. Isaacs, Character theory of finite groups, AMS Chelsea Publishing, Providence RI, 2011.

9. H. Koch, Classification of the primitive representations of the Galois group of local fields, Invent. Math. 40 (1977), 195-216.

10. M. Reeder, Adjoint Swan conductors I: the essentially tame case, Int. Math. Res. Not. IMRN (2017), doi: 10.1093/imrn/rnw301.

11. J.F. Rigby, Primitive linear groups containing a normal nilpotent subgroup larger than the centre of the group, J. London Math. Soc. 35 (1960), 389-400.

12. J-P. Serre, Corps locaux, Hermann, Paris, 1968.

King's College London, Department of Mathematics, Strand, London WC2R 2LS, UK.

E-mail address: colin.bushnell@kcl.ac.uk

Laboratoire de Mathématiques d'Orsay, Univ Paris-Sud, CNRS, Université Paris-Saclay, 91405 Orsay, France.

E-mail address: Guy.Henniart@math.u-psud.fr 